\title{
The Degrees of Freedom in Hot Quenched QCD
}

\author{
Sourendu Gupta ${ }^{\mathrm{a}}$ \\ ${ }^{a}$ HLRZ, c/o KFA Jülich, D-5170 Jülich, Germany
}

In quenched QCD, on lattices with $N_{\tau}=4$, the absence of a pole in the pseudoscalar meson channel for $T>T_{c}$ is demonstrated. A set of effective 4-fermi couplings is extracted. It is observed that this coupling is small in the vector channel for all $T>T_{c}$, but not in the pseudoscalar channel. The temperature dependence of hadronic parameters below $T_{c}$ is found to be small.

(Talk presented at the Lattice '92 conference, Amsterdam, September 1992.)

\section{Introduction}

We present computations of screening masses and couplings both above and below the finitetemperature phase transition in quenched QCD with $N_{\tau}=4$. We investigate the $\rho$ and the pion below $T_{c}$, extracting the meson masses as well as the pion decay constant $f_{\pi}$. We show that data indicate the absence of mesons above $T_{c}$. We extract effective couplings above $T_{c}$, and indicate how one can obtain lattice estimates for dimuon cross sections in the plasma phase.

We lay special emphasis on the measurement of mesonic susceptibilities

$\chi_{\Gamma}=G_{\Gamma}(0)$.

Here $\Gamma$ denotes spin-flavour quantum numbers, and $G(0)$ is the momentum-space correlator at zero four-momentum. If the low-lying excitations in the channel $\Gamma$ are bosonic, then

$\chi_{\Gamma} \sim m_{\Gamma}^{-\gamma / \nu}$,

where $m_{\Gamma}$ is the mass of the meson and the exponent is the usual ratio of anomalous dimensions. In free field theory this exponent has value 2 . Recall that, even in a purely bosonic theory, interactions give rise to a cut in addition to the pole (via loop corrections to the 2-point function) which is instrumental in changing $\gamma / \nu$ from its free field value. However, when the theory admits no pole in the channel $\Gamma$, the spectral representation of $g_{\Gamma}$ has only cuts, and $\chi_{\Gamma}$ goes to a constant as the screening mass is extrapolated to zero.
In order to construct the susceptibilities, we work with correlators in which spins and chiralities are projected out. These are constructed from the usual $S, P S, V T$ and $P V$ correlators by the methods of [1], and denoted by the $S U(2)$ flavour notation $\pi, \sigma, \rho$ and $A$ (although we use 4 -flavour staggered fermions).

The analysis of susceptibilities involves a variation of the screening mass (in lattice units) at fixed temperature. In the pion channel, this requires simply a variation of the quark mass at fixed $\beta$. In both the low and high temperature phases of the theory, a wide range of $\mu_{\pi}$ can be reached by this means. For a similiar variation of $\mu_{\rho}$, it is not sufficient to tune the quark mass; a variation of $\beta$ is required. Thus a study of the $\rho$ channel similiar to the analysis we present here for pions requires a more ambitious computation on a sequence of lattices with changing $N_{\tau}$ and $\beta$ but fixed $T$. We have not attempted to do this.

Our runs have been performed on lattices with $N_{\tau}=4$, and with one of the spatial sizes large $\left(16 \leq N_{z} \leq 32\right)$. Thus we have been able to follow correlations to distances upto $4 / T$. This is necessary if one wants to see possible nonperturbative effects at high temperatures. We have constructed meson screening correlators for several different quark masses $\left(0.01 \leq m_{q} \leq 0.15\right)$ in order to perform extrapolations to the chiral limit. In the low-temperature phase the square of the pion mass extrapolates to zero against $m_{q}$, whereas in all other cases the screening mass extrapolates linearly against $m_{q}$. Full details of the runs are available in [2]. 
Table 1

Screening masses in the chiral limit. A free fermion theory give a mass 1.32 for $N_{\tau}=4$.

\begin{tabular}{lllrr}
\hline \multicolumn{1}{r}{$\beta$} & $\mu_{\pi} a$ & $\mu_{\sigma} a$ & $\mu_{\rho} a$ & $\mu_{A} a$ \\
\hline 5.5 & $0.005 \pm 0.002$ & & $1.47 \pm 0.02$ & \\
5.75 & $0.786 \pm 0.002$ & $0.847 \pm 0.007$ & $1.34 \pm 0.01$ & $1.32 \pm 0.05$ \\
5.8941 & $1.05 \pm 0.01$ & $1.08 \pm 0.01$ & $1.33 \pm 0.03$ & $1.37 \pm 0.05$ \\
6.05 & $1.13 \pm 0.01$ & $1.14 \pm 0.02$ & $1.36 \pm 0.02$ & $1.38 \pm 0.04$ \\
\hline
\end{tabular}

\section{Results in the low-temperature phase}

Results below $T_{c}$ come from measurements on $4 \times 8^{2} \times 16$ and $4 \times 8^{2} \times 32$ lattices at $\beta=5.5$ (corresponding to $T=0.75 T_{c}$ ). There is little volume dependence in our results. Estimates of the screening masses in the zero quark-mass limit are given in Table 1. The $\sigma$ and $A$ masses are difficult to extract in this phase. The value of $m_{\rho}$ we obtain at $T=0.75 T_{c}$ is in agreement with the $T=0$ results of [3]. We find a massless pion, indicating broken chiral symmetry. This is confirmed by the $m_{q}=0$ extrapolation

$\langle\bar{\psi} \psi\rangle a^{3}= \begin{cases}0.227 \pm 0.006 & \left(N_{z}=16\right) \\ 0.231 \pm 0.005 & \left(N_{z}=32\right) .\end{cases}$

From fits of the form $\mu_{\pi}^{2} a^{2}=A_{\pi} m_{q} a+B_{\pi}$, we find

$A_{\pi}= \begin{cases}6.6 \pm 0.2 & \left(N_{z}=16\right) \\ 6.60 \pm 0.04 & \left(N_{z}=32\right),\end{cases}$

in complete consistency with the $T=0$ measurements of [3]. Assuming that the Gell-MannOakes-Renner identity holds also at finite temperature, we can extract the value of $f_{\pi}$ using the measured value of $\langle\bar{\psi} \psi\rangle$ and the slope $A_{\pi}$ obtained from the fits above. We find

$f_{\pi} a= \begin{cases}0.185 \pm 0.007 & \left(N_{z}=16\right) \\ 0.187 \pm 0.002 & \left(N_{z}=32\right) .\end{cases}$

This is smaller than the corresponding zero temperature measurement at the same coupling. The temperature dependence of $f_{\pi}$ comes entirely from that of $\langle\bar{\psi} \psi\rangle$, since $A_{\pi}$ is temperature independent.

We find a physical pion state giving rise to a pole in the spectral function. On the lattice this manifests itself as a $1 / \mu \sinh \mu$ dependence of $\chi$ on the measured screening mass. In fact, a fit of the form $1 / 2 \chi=A \mu \sinh \mu+B$ yields

$$
B= \begin{cases}0.0008 \pm 0.0004 & \left(N_{z}=16\right) \\ 0.0008 \pm 0.0003 & \left(N_{z}=32\right) .\end{cases}
$$

Finite-volume effects are invisible, being entirely within the error bars.

\section{Results in the high-temperature phase}

These results come from simulations on $4 \times$ $8^{2} \times 16,4 \times 12^{2} \times 24$ and $4 \times 16^{2} \times 32$ lattices at $\beta=5.75,5.8941$ and 6.05 (corresponding respectively to $1.1,1.5$ and $2 T_{c}$ ). Our measurements of the screening masses (shown in Table 1) at high temperatures are consistent with earlier measurements in the quenched theory [4]. The $\rho$ and $A$ screening masses are in good agreement

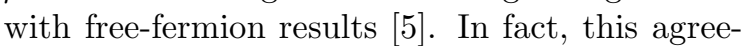
ment extends beyond the screening mass to the full correlation function.

Susceptibility measurements strongly indicate the absence of a pole in the pion spectral density above $T_{c}$. At $\beta=5.75$, a fit of the form $1 / 2 \chi=$ $A \mu \sinh \mu+B$ yields

$B= \begin{cases}0.041 \pm 0.003 & \left(4 \times 12^{2} \times 24 \text { lattice }\right) \\ 0.043 \pm 0.004 & \left(4 \times 16^{2} \times 32 \text { lattice }\right) .\end{cases}$

Table 2

Effective 4-fermi couplings.

\begin{tabular}{rlrr}
\hline Lattice & $\beta$ & $g_{\gamma_{5}} T^{2}$ & $g_{\gamma_{\mu}} T^{2}$ \\
\hline $4 \times 8^{2} \times 16$ & 5.75 & $1.19 \pm 0.03$ & $0.21 \pm 0.03$ \\
& 5.8941 & $0.61 \pm 0.04$ & $0.03 \pm 0.04$ \\
& 6.05 & $0.38 \pm 0.02$ & $-0.02 \pm 0.03$ \\
$4 \times 12^{2} \times 24$ & 5.75 & $1.10 \pm 0.05$ & $0.14 \pm 0.04$ \\
& 6.05 & $0.45 \pm 0.02$ & $0.03 \pm 0.03$ \\
$4 \times 16^{2} \times 32$ & 5.75 & $1.19 \pm 0.06$ & $0.21 \pm 0.05$ \\
& 6.05 & $0.39 \pm 0.03$ & $-0.03 \pm 0.03$ \\
\hline
\end{tabular}


Thus, a pion pole is ruled out to $10 \sigma$. Finite-size effects are strong on our smallest lattice, but not on the two larger volumes quoted above.

In the absence of bosonic poles, the chiral symmetry of the theory must be represented in terms of an effective fermionic theory. The general form of such an action is

$S_{\text {eff }}=\int_{x} \bar{\psi} D \psi+\sum g_{\Gamma}(\bar{\psi} \Gamma \psi)^{2}$,

where $D$ is the Dirac operator, $g_{\Gamma}$ are effective 4fermi couplings, and $\Gamma$ are spin-flavour projectors. We define the effective couplings by summing ladder graphs for $\chi_{\Gamma}$, and inverting the relation to obtain $g_{\Gamma}$. The results of these measurements are listed in Table 2. Parity related couplings are equal. It is an interesting cross-check that, in the context of such 4 -fermi theories, the derived values of the couplings require a chirally symmetric vacuum. This effective action thus summarises the information contained in the measurement of hadronic correlators for $T>T_{c}$. It remains to be checked whether the 'spatial wavefunctions' reported in [6] can be accomodated in this picture. Just as a $S U\left(N_{f}\right) \times S U\left(N_{f}\right)$ chiral model provides an effective theory for low energy QCD for $T<T_{c}$, the 4 -fermi theory with the couplings given here can be used to examine low-energy processes at $T>T_{c}$. In the absence of mesonic poles in correlations of currents bilinear in the quark field, it becomes possible to measure many interesting rate processes on the lattice. An example is provided by the rate for low-mass dimuon production. This is obtained from a vector correlator. This spatial correlation function can be measured on the lattice and Wick-rotated using the information obtained here on the spectral density function.

\section{REFERENCES}

1. J. P. Gilchrist, G. Schierholz, H. Schneider and M. Teper, Nucl. Phys., B248 (1984) 29.

2. Sourendu Gupta, Phys. Lett., B288 (1992) 171.

3. M. Fukugita, S. Ohta, Y. Oyanagi and A. Ukawa, Phys. Lett., 191B (1987) 164.

4. A. Gocksch, P. Rossi and U. M. Heller, Phys. Lett., B205 (1988) 334.
5. K. Born et al., Phys. Rev. Lett., 67 (1991) 302 ;

V. L. Eletskii and B. L. Ioffe, Sov. J. Nucl. Phys., 48 (1988) 384.

6. C. Bernard et al., Phys. Rev. Lett., 68 (1992) 2125 . 